\title{
Collisional and radiative effects in the state-selective preparation of translationally cold molecular ions in ion traps
}

\author{
Xin Tong, Dieter Wild, and Stefan Willitsch* \\ Department of Chemistry, University of Basel, Klingelbergstrasse 80, CH-4056 Basel, Switzerland
}

(Received 14 December 2010; published 18 February 2011)

\begin{abstract}
We give a detailed characterization of a recently developed method to prepare translationally cold, internally state-selected molecular ions in ion traps [X. Tong, A. H. Winney, and S. Willitsch, Phys. Rev. Lett. 105, 143001 (2010)]. The technique relies on the generation of molecular ions in a well-defined rotational-vibrational quantum state using threshold photoionization followed by sympathetic cooling of the translational motion with laser-cooled $\mathrm{Ca}^{+}$ions. We discuss the experimental requirements for the successful generation and sympathetic cooling of state-selected ions, explore the influence of collisional and radiative processes on the population redistribution dynamics, and give an assessment of the scope of the method.
\end{abstract}

DOI: 10.1103/PhysRevA.83.023415

PACS number(s): 37.10.-x, 34.50.Cx, 52.27.Jt

\section{INTRODUCTION}

Over the past few years, impressive progress has been made in the preparation and control of the internal quantum state of "ultracold" molecules at very low translational temperatures, $T_{\text {trans }} \ll 1 \mathrm{~K}$ [1-6]. Neutral diatomic molecules synthesized from ultracold atomic gases have been prepared in specific rotational, vibrational, and even hyperfine quantum states, demonstrating full control over the most important molecular degrees of freedom. Such quantum-state and kinetic-energy controlled molecules are of considerable interest for a number of applications, including quantum-information processing [7] and the study of collisional and chemical processes in the ultracold regime [8-10].

Similar efforts have also been undertaken to control the internal state of translationally cold molecular ions in ion traps. The translational motion of molecular ions can be cooled sympathetically using the Coulomb interaction with simultaneously trapped, laser-cooled atomic ions [11,12]. In this way, translational temperatures in the millikelvin regime can readily be achieved. Under these conditions, the ions localize in the ion trap to form ordered structures known as "Coulomb crystals". However, in contrast to buffer-gas cooling methods in cryogenic traps [13], sympathetic cooling does not affect the internal degrees of freedom, i.e., the rotational and vibrational motion of the molecular ions. As a consequence, translationally cold, but internally warm, samples of ions are obtained in which the internal-state populations are usually in thermal equilibrium with the ambient blackbody radiation (BBR) field [14,15].

Two different approaches have recently been implemented to overcome this problem. Over the past years, Drewsen and co-workers have proposed several schemes which rely on optical pumping to modify the initially broad distribution of populations in sympathetically cooled molecular ions in order to achieve a higher degree of state purity [16-18]. A particularly simple scheme is based on optical pumping on one or several selected rovibrational transitions, aided by a redistribution of the population using BBR in order to accumulate population in a well-defined rotational level [16].

*stefan.willitsch@unibas.ch
This approach has recently been implemented experimentally by Staanum et al. [19] and Schneider et al. [20], who were able to increase the population in the rotational ground state to $37 \%$ in $\mathrm{MgH}^{+}$and $78 \%$ in $\mathrm{HD}^{+}$, respectively.

An alternative method for the internal-state preparation of translationally cold molecular ions has recently been demonstrated by our group [21]. Our approach is based on the initial state-selective generation of the molecular ions using threshold photoionization followed by sympathetic cooling of the translational motion. As discussed in our previous paper [21], we achieved a population in the rovibronic ground state of $93 \pm 11 \%$ after sympathetic cooling using molecular nitrogen ions $\left(\mathrm{N}_{2}{ }^{+}\right)$as a test system. Similar state purities were achieved in excited rotational states.

In this paper, we report a detailed characterization of our method in addition to the results presented in our previous paper [21]. We give a comprehensive description of the experimental procedure, outline the requirements for the successful generation and sympathetic cooling of state-selected molecular ions, explore the applicability of the method to different systems (in particular polar ions), and discuss in detail radiative and collisional processes affecting the lifetime of the initially prepared quantum state.

\section{GENERATION OF STATE-SELECTED MOLECULAR IONS}

Over the past decades, photoionization has emerged as one of the most important techniques to produce molecular ions in specific quantum states. Resonance-enhanced multiphoton ionization (REMPI) has been employed to generate molecular cations in selected vibrational levels by taking advantage of near-diagonal Franck-Condon factors in the ionization through vibrationally excited Rydberg states (see, e.g., Refs. [22,23] and references cited therein).

Rotational-state selection is more difficult to achieve. REMPI experiments are conventionally performed by exciting out of a selected intermediate state deep into the ionization continuum, as illustrated in Fig. 1(a). Under these conditions, the cation is usually generated in a narrow distribution of rotational states (even if the molecule is ionized out of a single rotational level) in line with rotational photoionization 

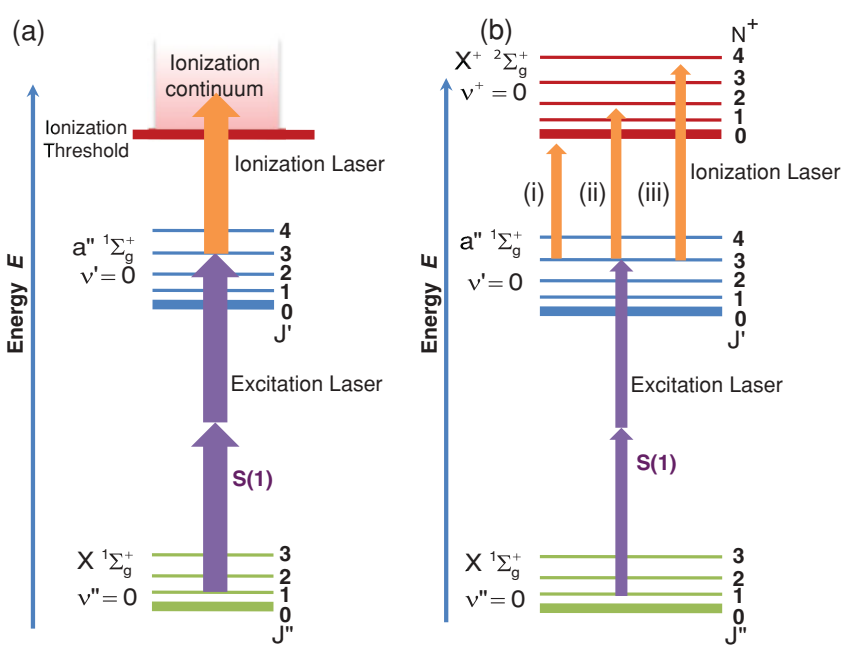

FIG. 1. (Color online) (a) Resonance-enhanced $\left[2+1^{\prime}\right]$ photon ionization scheme in molecular nitrogen. Ionization is performed by exciting deep into the ionization continuum leading to a narrow distribution of cationic rotational levels. (b) Resonance-enhanced $\left[2+1^{\prime}\right]$ photon threshold-ionization scheme via selected rotational levels of the $a^{\prime \prime}{ }^{1} \Sigma_{g}^{+}, v^{\prime}=0$ state. By ionizing only slightly above the lowest rotational-ionization threshold accessible from a selected intermediate level, molecular ions can be produced in a single rotational quantum state.

selection and propensity rules [24-27]. Within the framework of the orbital ionization model, the distribution of cationic rotational levels can be predicted in terms of the coupling of the rotational angular momentum to an electronic angularmomentum "hole" generated in the molecular core by the removal of the photoelectron (see Ref. [26] for details).

Complete rotational- and vibrational-state selection can be achieved by pulsed-field ionization (PFI) of high Rydberg states located just below the relevant rovibrational ionization threshold [28-30]. However, to achieve optimal state selectivity in field ionization, well-defined homogeneous electric fields are required [31], which are incompatible with a rf ion trap fundamentally relying on inhomogeneous fields to trap charged particles [12]. As an alternative, rotational-state selection in the cation can be achieved by exciting into the ionization continuum located slightly above the lowest rotational ionization threshold accessible from a well-defined rotational state in the neutral by photoionization selection and propensity rules. In this process, the ionization out of a single rotational level can be ensured by a suitable resonanceenhanced excitation scheme. In contrast to PFI, this all-optical approach for the generation of state-selected ions can readily be implemented in an ion trap.

In the present study, molecular nitrogen ions were produced in a selected rovibronic quantum state using a twocolor $\left[2+1^{\prime}\right]$ resonance-enhanced threshold-photoionization scheme. Ionization was carried out via selected rotational levels of the $a^{\prime \prime}{ }^{1} \Sigma_{g}^{+}, v^{\prime}=0$ electronic state of $\mathrm{N}_{2}$, which was excited by two photons from the electronic ground state [32] [see Fig. 1(b)]. Here $v$ denotes the vibrational quantum number and the superscripts ", ', and + stand for the neutral ground, neutral excited, and ionic ground electronic states. Previous rotationally resolved photoelectron studies [29] have

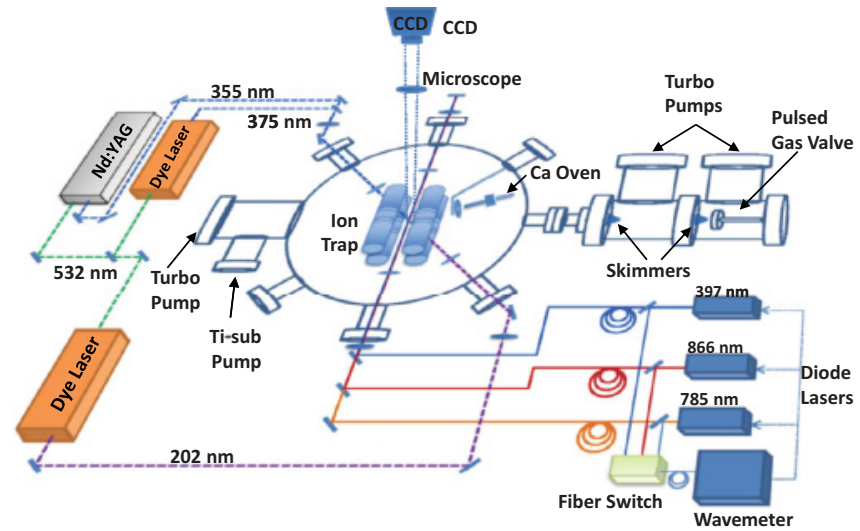

FIG. 2. (Color online) Schematic of the experimental setup.

established a strong $\Delta N=N^{+}-J^{\prime}=0$ rotational propensity rule for photoionization out of the $a^{\prime \prime}{ }^{1} \Sigma_{g}^{+}$state $\left(N^{+}\right.$and $J^{\prime}$ denote the rotational quantum numbers in the cationic and intermediate states, respectively). This propensity rule can be explained by the dominant $s$ character of the molecular orbital from which ionization occurs [26]. Ionizing transitions with $|\Delta N|=2,4$ bear considerably weaker intensity and transitions with $\Delta N=$ odd are forbidden on grounds of the conservation of nuclear spin symmetry. Thus, by setting the wave number of the ionization laser only slightly above the lowest rotational ionization thresholds accessible from $J^{\prime}, \mathrm{N}_{2}{ }^{+}$ions in well-defined rotational levels can be produced with very high selectivity. In our previous paper, we demonstrated the efficacy of this scheme for the production of $\mathrm{N}_{2}{ }^{+}$ions in the $N^{+}=0$ and $N^{+}=3$ rotational levels [21].

\section{EXPERIMENT}

Figure 2 shows a schematic of the experimental setup used in the present study. The apparatus consists of two sets of vacuum systems: an ultrahigh-vacuum (UHV) chamber housing a linear Paul ion trap and a source to generate molecular beams of neutral precursor molecules for the ions.

\section{A. Ion trap}

The linear Paul ion trap used in the present study consists of four cylindrical electrodes arranged in a quadrupolar configuration, as shown schematically in Fig. 2 and as described in Ref. [12]. Radio frequency (rf) voltages of alternating polarity between adjacent rods are applied to create time-varying, inhomogeneous electric fields, which enable a confinement of the ions in the plane perpendicular to the longitudinal trap axis. Typical $\mathrm{rf}$ amplitudes and frequencies used are $V_{\mathrm{rf}, 0-\mathrm{p}}=120 \mathrm{~V}$ and $\Omega_{\mathrm{rf}}=2 \pi \times 3.2 \mathrm{MHz}$, respectively. Each of the four electrodes is sectioned into three parts to enable the application of additional dc voltages, $V_{\mathrm{END}}=1-5 \mathrm{~V}$, to eight endcap segments for trapping ions along the trap axis. The diameter of the electrodes is $8.0 \mathrm{~mm}$ and the distance from the trap center to the surface of the rods is $3.5 \mathrm{~mm}$.

\section{B. Photoionization}

To preserve the originally prepared state of the molecular ions during and after the sympathetic-cooling process, it 
proved essential to separate the production and cooling regions of the ions (see Sec. IV C). Consequently, the ions were generated in a narrowly collimated molecular beam (with diameter $250 \mu \mathrm{m}$ ) traversing the ion trap at a distance of $\approx 400 \mu \mathrm{m}$ from the trap center, where they localized after sympathetic cooling. The molecular-beam assembly was mounted on a horizontal translation stage, enabling the alignment of the beam trajectory through the ion trap with a precision of $\approx 10 \mu \mathrm{m}$. A beam of internally cold $\mathrm{N}_{2}$ molecules was created in an adiabatic supersonic expansion of the pure gas into high vacuum through a piezoelectric gas valve. The gas nozzle, with an orifice diameter of $0.8 \mathrm{~mm}$, operated at a repetition rate of $10 \mathrm{~Hz}$, a pulse duration of $1 \mathrm{~ms}$, and a backing pressure of 1.5 bars. Two skimmers, with apertures of 0.4 and $0.2 \mathrm{~mm}$, served to collimate the molecular beam and acted as differential pumping stages to separate the UHV region from the molecular-beam source. During operation, the pressure in the ion trap chamber was maintained at $p=5 \times$ $10^{-10}$ mbar.

Resonance-enhanced $\left[2+1^{\prime}\right]$ threshold photoionization of $\mathrm{N}_{2}$ was carried out using the frequency-tripled $\left(\lambda_{1} \approx 202 \mathrm{~nm}\right)$ and -doubled ( $\lambda_{2} \approx 375 \mathrm{~nm}$ ) output of two Nd:YAG-pumped pulsed dye lasers (with bandwidth of the fundamental laser beam $\leqslant 0.2 \mathrm{~cm}^{-1}$ ). The 202 and $375 \mathrm{~nm}$ laser beams were operated at pulse energies of 0.2 and $1.5 \mathrm{~mJ}$, respectively, and were focused into the molecular beam using lenses with focal lengths of 75 and $50 \mathrm{~cm}$, respectively. Under these conditions, ensembles of 20-30 sympathetically cooled, state-selected $\mathrm{N}_{2}^{+}$ions were obtained after a loading time of typically 20-30 s.

\section{Ion cooling}

The state-selected $\mathrm{N}_{2}^{+}$ions were sympathetically cooled to millikelvin secular temperatures using the Coulomb interaction with simultaneously trapped laser-cooled ${ }^{40} \mathrm{Ca}^{+}$ions [12]. The $\mathrm{Ca}^{+}$ions were produced inside the trap by nonresonant photoionization from an atomic beam of calcium emanating from an oven at a temperature of $\approx 600 \mathrm{~K}$. Doppler laser cooling of $\mathrm{Ca}^{+}$on the $(4 s)^{2} S_{1 / 2} \rightarrow(4 p)^{2} P_{1 / 2}$ transition was performed using two counterpropagating diode-laser beams at $397 \mathrm{~nm}$ with a total power of $0.8 \mathrm{~mW}$ per beam focused to $\approx 180 \mu \mathrm{m}$ in the trap center. A laser beam at $866 \mathrm{~nm}$ ( $0.4 \mathrm{~mW}$ focused to $\approx 250 \mu \mathrm{m}$ in the trap center) served to repump population from the $(3 d)^{2} D_{3 / 2}$ state in order to close the laser-cooling cycle. The frequencies of both laser beams were regulated by a high-accuracy $(<30 \mathrm{MHz})$ wavelength meter (HighFinesse WSU/30). The resulting Coulomb crystals were imaged by collecting the spatially resolved fluorescence of the $\mathrm{Ca}^{+}$ions using a charge-coupled device (CCD) camera coupled to a microscope (with magnification $16 \times$ ). Because of their lighter mass in comparison to $\mathrm{Ca}^{+}$, the sympathetically cooled $\mathrm{N}_{2}{ }^{+}$ions localize in the center of the ion trap and appear as a dark, nonfluorescing core in the middle of the crystals (see, e.g., Fig. 4).

In the present experiments, the central region of Coulombcrystallized $\mathrm{N}_{2}^{+}$ions showed an asymmetric shape because of radiation pressure on the $\mathrm{Ca}^{+}$ions. As discussed in our previous paper [21], the precise appearance of the dark core region sensitively depends on the numbers of sympathetically cooled ions. This number can accurately be established by a comparison with simulated fluorescence images obtained from molecular-dynamics (MD) calculations containing a well-defined number of ions. Moreover, a comparison with simulated images at different secular temperatures allows an accurate determination of the ion kinetic energies [33,34]. In our experiments, the secular temperatures of the laser-cooled $\mathrm{Ca}^{+}$and sympathetically cooled $\mathrm{N}_{2}^{+}$ions were established to be $8 \pm 1$ and $11 \pm 1 \mathrm{mK}$, respectively.

\section{Population diagnostics}

The rotational-state populations of the sympathetically cooled $\mathrm{N}_{2}^{+}$ions were probed using laser-induced chargetransfer (LICT) with Ar atoms [21,35]. Vibrationally excited $\mathrm{N}_{2}{ }^{+}$ions react with $\mathrm{Ar}$ according to $\mathrm{N}_{2}^{+}\left(v^{+} \geqslant 1\right)+\mathrm{Ar} \rightarrow$ $\mathrm{N}_{2}+\mathrm{Ar}^{+}$. On the other hand, for $\mathrm{N}_{2}^{+}$ions originally produced in the $v^{+}=0$ vibrational level, the reaction is endothermic and thus suppressed under our experimental conditions. To probe the population in different spin-rotational levels of the vibrational ground state, the $\mathrm{N}_{2}^{+}$ions were optically excited to the $A^{+2} \Pi_{u}, v=2$ state using $15 \mathrm{~mW}$ of diode-laser radiation in the wavelength range from 783 to $787 \mathrm{~nm}$. Depending on the spin-rotational component $F_{1}, F_{2}$ accessed in the $X^{+}$ ${ }^{2} \Sigma_{\mathrm{g}}^{+}, v^{+}=0$ state (Hund's coupling case $b$ ), excitations to the corresponding ${ }^{2} \Pi_{u, 3 / 2}$ or ${ }^{2} \Pi_{u, 1 / 2}$ components of the $A^{+}$state (Hund's coupling case $a$ ) were used in line with spin-selection rules (see Fig. 3 and Ref. [36]). The excitation of the relevant transitions was ensured by continuously scanning the laser frequency in a window of $1.0 \mathrm{GHz}$ over the line positions tabulated in Ref. [37].

Subsequent fluorescent decay of the electronically excited state populates vibrationally excited levels in the $X^{+}{ }^{2} \Sigma_{g}^{+}$ ground electronic state with a probability of 55\% [35]. This number thus defines the maximum proportion of $\mathrm{N}_{2}^{+}$ions

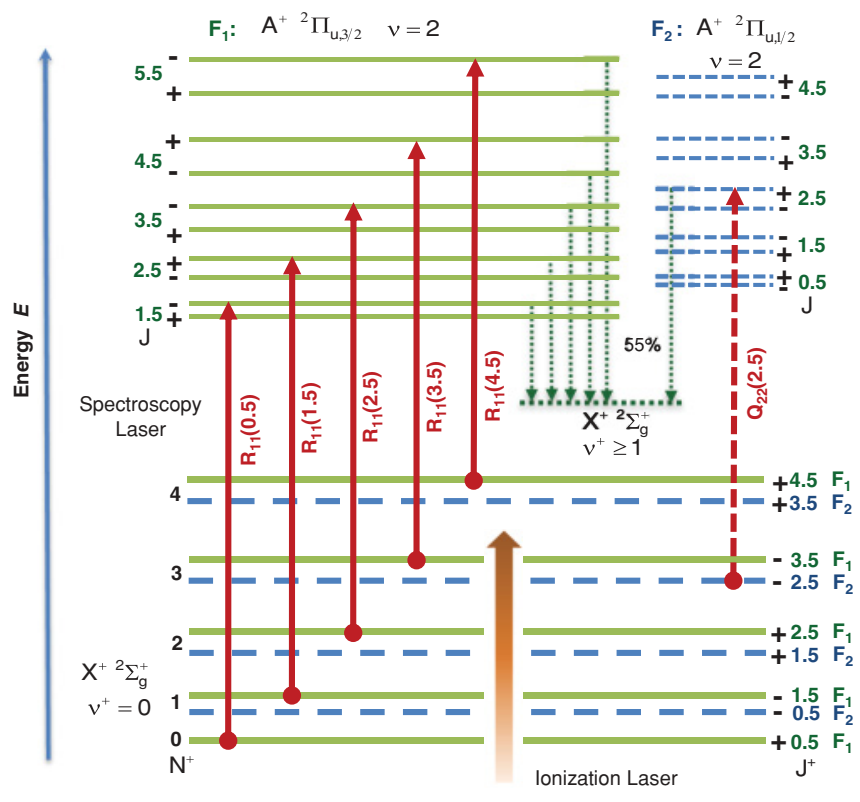

FIG. 3. (Color online) Laser-induced charge-transfer (LICT) scheme to probe the population of the different spin-rotational levels in the $X^{+2} \Sigma_{\mathrm{g}}^{+}, v^{+}=0$ state of $\mathrm{N}_{2}^{+}$. 


$\begin{gathered}\text { Excitation Energy } \\ \text { (offset from } \mathrm{S}(1) \text { transition) }\end{gathered}$
$\begin{gathered}\text { Experimental bi-component } \\ \text { Coulomb crystal image } \\ \left(-2.4 \mathrm{~cm}^{-1}\right)\end{gathered}$
$98859.0 \mathrm{~cm}^{-1}$
$\left(-1.2 \mathrm{~cm}^{-1}\right)$
$98860.2 \mathrm{~cm}^{-1}$
$\left(0 \mathrm{~cm}^{-1}\right)$
$98861.4 \mathrm{~cm}^{-1}$
$\left(+1.2 \mathrm{~cm}^{-1}\right)$

$98862.6 \mathrm{~cm}^{-1}$
$\left(+2.4 \mathrm{~cm}^{-1}\right)$

FIG. 4. Fluorescence images of $\mathrm{Ca}^{+} / \mathrm{N}_{2}{ }^{+}$bicomponent Coulomb crystals. The $\mathrm{N}_{2}^{+}$ions were produced by a $\left[2+1^{\prime}\right]$ resonanceenhanced multiphoton ionization (REMPI) process in the region of the $X^{1} \Sigma_{g}^{+}, v^{\prime \prime}=0 \rightarrow a^{\prime \prime}{ }^{1} \Sigma_{g}^{+}, v^{\prime}=0 \mathrm{~S}(1)$ two-photon transition. The sympathetically cooled molecular ions are visible as a nonfluorescing region in the center of the crystals. The maximum number of sympathetically cooled $\mathrm{N}_{2}^{+}$ions could be observed on resonance (zero offset) from the $\mathrm{S}(1)$ transition indicating the enhancement in the photoionization yield through selection of the $J^{\prime}=3$ intermediate rotational level.

that can undergo charge transfer. Charge transfer was initiated immediately after laser excitation by introducing argon gas at a pressure $p(\mathrm{Ar})=5 \times 10^{-8}$ mbar into the chamber through a leak valve. The $\mathrm{Ar}^{+}$ions produced by LICT remain trapped and are sympathetically cooled into the Coulomb crystal. However, because of the almost identical masses, the $\mathrm{Ar}^{+}$ions diffuse among the $\mathrm{Ca}^{+}$ions in the Coulomb crystal and cannot be observed in the images. Consequently, the removal of $\mathrm{N}_{2}^{+}$ions from the central core as a consequence of LICT can directly be detected in the images, and the proportion of removed $\mathrm{N}_{2}^{+}$ ions represents a measure of the population in the relevant spin-rotational level.

\section{RESULTS}

\section{A. Threshold photoionization of $\mathbf{N}_{2}$}

The successful generation of rotationally state-selected ions was already demonstrated in our previous paper [21]. Here, we characterize in detail two key requirements for a state-selective $\left[2+1^{\prime}\right]$ threshold-photoionization scheme: the ability to precisely select a well-defined rotational intermediate level and the suppression of unselective photoionization processes, in particular one-color $[2+1]$ ionization. In this context, it proved essential to carefully balance the intensities of the excitation and ionization lasers as described in Sec. III B.

To ensure the selection of a well-defined intermediate rotational level, $\left[2+1^{\prime}\right]$ REMPI experiments were performed, as shown schematically in Fig. 1(a). The total ionization wave number was set to $\geqslant 80 \mathrm{~cm}^{-1}$ above the lowest ionization threshold, providing enough excess energy to ensure a constant ion yield while scanning the frequency of the excitation laser over the rotational resonances of the $a^{\prime \prime}{ }^{1} \Sigma_{g}^{+}, v^{\prime}=0$ state. Figure 4 shows the results of an experiment in which the two-photon, excitation-laser wave number was scanned from 98857.8 to $98862.6 \mathrm{~cm}^{-1}$, with increments of $1.2 \mathrm{~cm}^{-1}$, over the $\mathrm{S}(1)$ transition (corresponding to the excitation $\left.X^{1} \Sigma_{g}^{+}, v^{\prime \prime}=0, J^{\prime \prime}=1 \rightarrow a^{\prime \prime}{ }^{1} \Sigma_{g}^{+}, v^{\prime}=0, J^{\prime}=3\right)$. Molecular ions were produced from the molecular beam for a period of $25 \mathrm{~s}$ and subsequently cooled into a $\mathrm{Ca}^{+}$Coulomb crystal. Under these conditions, the maximum number of sympathetically cooled ions could be observed at an excitation wave number of $2 \tilde{v}_{1}=98860.2 \mathrm{~cm}^{-1}$, corresponding to the center of the $S(1)$ line reported in Ref. [32]. Because adjacent rotational lines in the $S$ branch are separated by $\approx 7.5 \mathrm{~cm}^{-1}$ [32], we expect the state contamination by the simultaneous excitation of neighboring lines to be negligible, and conclude that only the $J^{\prime}=3$ level was selected in the excitation step.

Figure 5 shows a series of experiments in which the wave number of the ionization laser was scanned over successive rotational-ionization thresholds out of the $J^{\prime}=3$ intermediate level [as shown schematically in Fig. 1(b), (i)-(iii)]. The intensities observed in the rotationally resolved photoelectron spectrum in Ref. [29] reveal the important ionization channels out of this state. The dominant channel leads to the formation of $\mathrm{N}_{2}{ }^{+}$ions in $N^{+}=3$; two other channels resulting in the production of ions in $N^{+}=1$ and 5 carry considerably weaker intensity.

In the experiment shown in Fig. 5(a), the $\left[2+1^{\prime}\right]$ ionization wave number was set below the lowest ionization threshold of $\mathrm{N}_{2}$, which corresponds to the formation of $\mathrm{N}_{2}^{+}$in the rotational ground state, $N^{+}=0$ [see Fig. 1(b), (i)]. Under these conditions, the total $\left[2+1^{\prime}\right]$ photon energy is insufficient to photoionize $\mathrm{N}_{2}$. Indeed, after an ionization period of $30 \mathrm{~s}$,

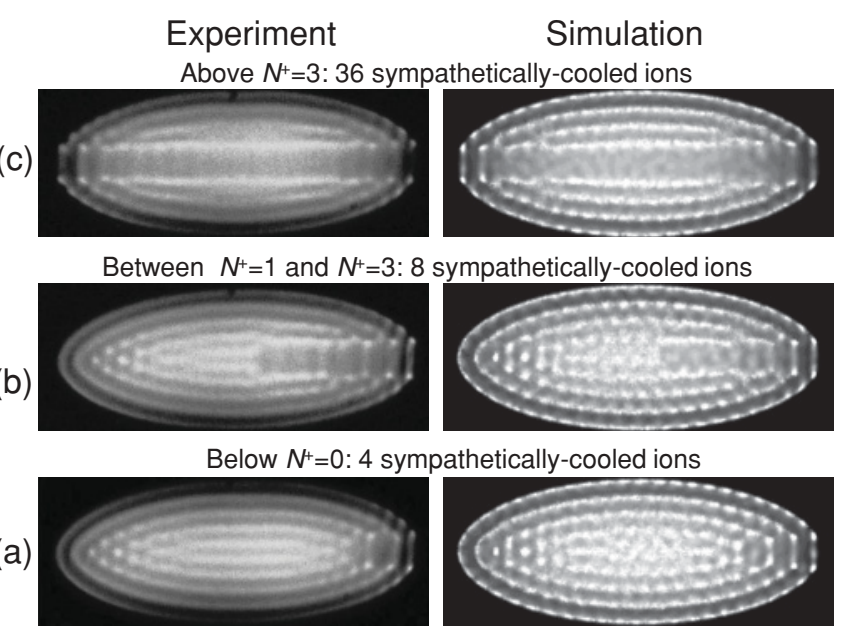

FIG. 5. Sympathetic cooling of $\mathrm{N}_{2}^{+}$ions produced by threshold photoionization via the $J^{\prime}=3$ intermediate rotational level. The frequency of the ionization laser was set (a) below the $N^{+}=0$ threshold, (b) between the $N^{+}=1$ and $N^{+}=3$ thresholds, and (c) lightly above the $N^{+}=3$ threshold. 
only $4 \pm 1$ sympathetically cooled ions were observed in the Coulomb-crystal core. Because the number of ions produced under these conditions did not vary when the $\mathrm{N}_{2}$ molecular beam was switched off, we infer that these ions are formed by nonresonant multiphoton ionization of background gas molecules.

By setting the two-color ionization wave number at $12 \mathrm{~cm}^{-1}$ above the $N^{+}=0$ threshold in between the $N^{+}=1$ and the $N^{+}=3$ thresholds, as illustrated in Fig. 1(b), (ii), $8 \pm 1$ sympathetically cooled ions could be observed in the Coulomb-crystal core [Fig. 5(b)]. We attribute $\approx 50 \%$ of these to the formation of $\mathrm{N}_{2}^{+}$ions in $N^{+}=1$ from ionization on the weakly allowed $J^{\prime}=3 \rightarrow N^{+}=1$ channel [29]. The remaining $50 \%$ are attributed to ions of background-gas molecules, the formation efficiency of which is expected to vary only insignificantly in the wave number interval studied. Finally, by setting the ionization wave number $8.8 \mathrm{~cm}^{-1}$ above the $N^{+}=3$ threshold [see Fig. 1(b), (iii)], the ionization yield increased markedly and $36 \pm 1$ sympathetically cooled ions were observed in the center of the Coulomb crystal [Fig. 5(c)]. We attribute $\approx 28$ of these to $\mathrm{N}_{2}^{+}$ions generated on the strong $J^{\prime}=3 \rightarrow N^{+}=3$ ionizing transition.

In conclusion, the results presented in Figs. 4 and 5 demonstrate both the high state selectivity of the $\left[2+1^{\prime}\right]$ threshold-ionization scheme and the minimization of unselective ionization processes under the present experimental conditions.

\section{B. Spin-rotational state populations}

For results on the population diagnostics of ions prepared in the $N^{+}=0$ and $N^{+}=3$ rotational states, we refer to our previous paper [21]. For completeness, we show in Fig. 6

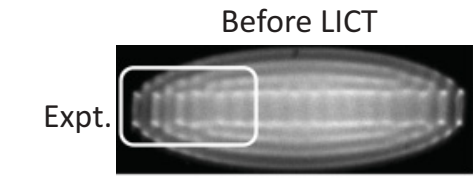

$F_{1}$
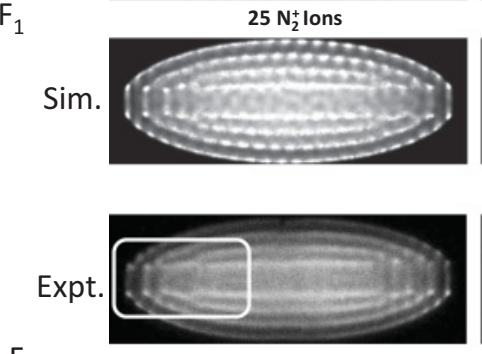

$\mathrm{F}_{2}$
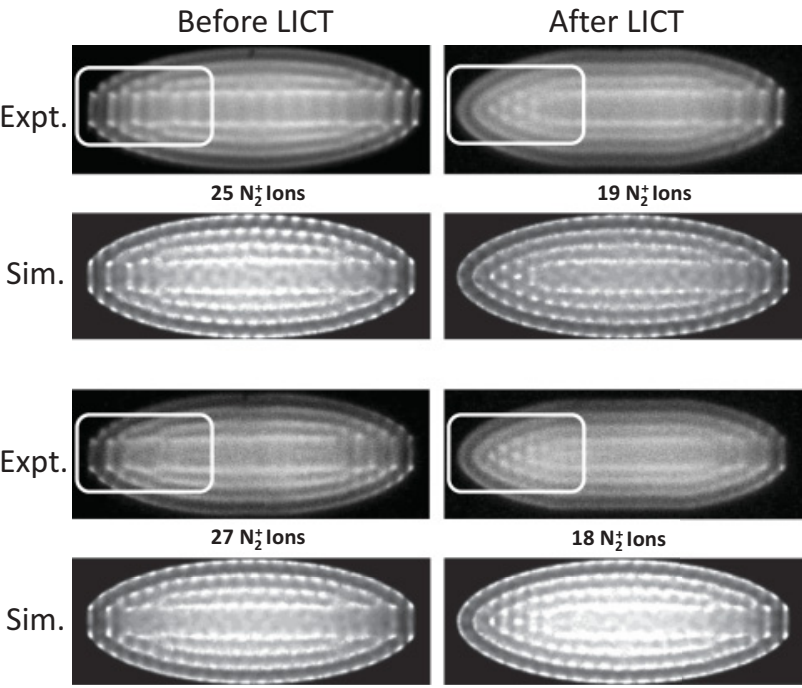

FIG. 6. Experiment (Expt.): Laser-induced charge transfer (LICT) experiments probing the populations in the $F_{1}$ and $F_{2}$ spin-rotational levels of the $N^{+}=3$ level following sympathetic cooling of $\mathrm{N}_{2}^{+}$ions produced by ionization above the $N^{+}=3$ threshold. Simulations (Sim.): The corresponding simulated images. The change in the number of sympathetically cooled $\mathrm{N}_{2}^{+}$ions as a consequence of LICT can be observed in the regions highlighted in the images. results of LICT experiments probing the population in the two spin-rotation components, $F_{1}$ and $F_{2}$, of the $N^{+}=3$ state produced by threshold ionization via $J^{\prime}=3$ and the corresponding simulations. The spectroscopic excitations used in the LICT step are indicated in Fig. 3. Following excitation out of the $N^{+}=3, F_{1}$ and $F_{2}$ components, we observe the removal of $24 \%(6 \pm 1$ out of $25 \pm 1)$ and $33 \%(9 \pm 1$ out of $27 \pm 1$ ) of the sympathetically cooled ions by charge transfer, respectively. Averaged over five experiments, the efficiencies for LICT out of both sublevels agree within the measurement uncertainties $\left(27 \pm 7 \%\right.$ and $28 \pm 7 \%$ for $F_{1}$ and $F_{2}$, respectively), indicating that both components are produced with equal probability using the current photoionization scheme. The total measured LICT efficiency out of the $N^{+}=3$ state amounts to $55 \pm 9 \%$, in agreement with the maximum value of $55 \%$ [21].

Figure 7 shows control experiments on the $\mathrm{R}_{11}(0.5)$, $\mathrm{R}_{11}(1.5), \mathrm{R}_{11}(2.5), \mathrm{R}_{11}(3.5)$, and $\mathrm{R}_{11}(4.5)$ transitions to probe the population in the $F_{1}$ spin-rotational component of the $N^{+}=0,1,2$, and 4 states (see Fig. 3). No evidence of population in these rotational levels was observed within our uncertainty limits. This result further confirms that the initially prepared rotational state $N^{+}=3$ is fully preserved during the sympathetic-cooling process. Moreover, we note that we observe no evidence for population in the $N^{+}=1$ state produced by ionization on the weakly allowed $J^{\prime}=$ $3 \rightarrow N^{+}=1$ channel. On the basis of the ionization studies discussed in Sec. IV A, we expect that less than two $\mathrm{N}_{2}^{+}$ions are produced in the $F_{1}$ component of $N^{+}=1$ under the present conditions, which is below the limit of the detection sensitivity of the present LICT scheme.

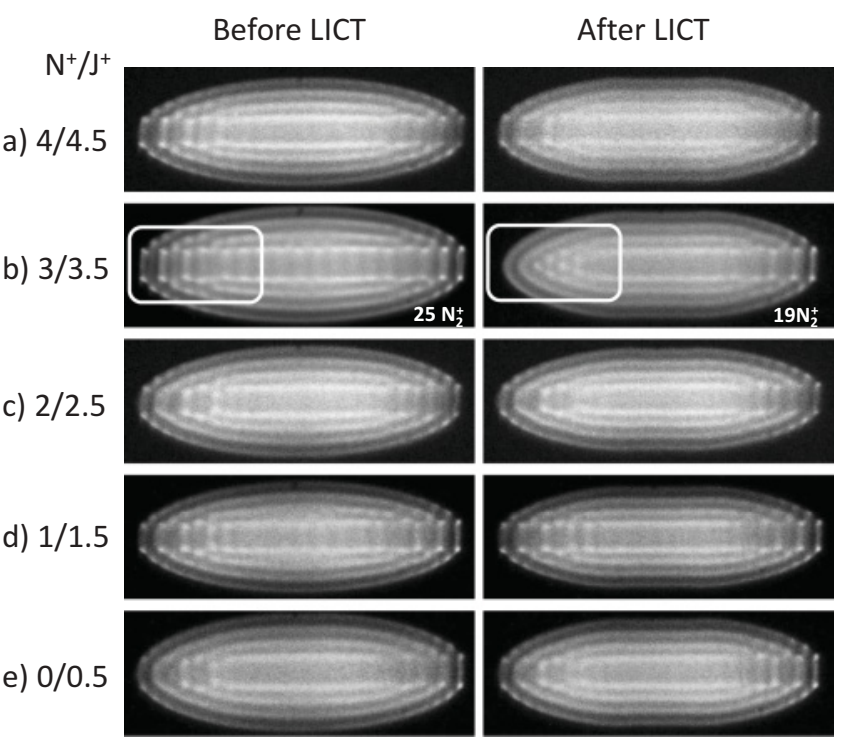

FIG. 7. LICT experiments probing the population in the $F_{1}$ spinrotational component of different rotational levels after sympathetic cooling of $\mathrm{N}_{2}^{+}$ions produced via threshold ionization above the $N^{+}=3$ threshold. LICT could only be observed following excitation from the $N^{+}=3$ level as seen from a comparison of the highlighted regions in (b). These results demonstrate the high rotational selectivity of the present threshold-photoionization scheme and the conservation of the population in the originally prepared state during sympathetic cooling. 


\section{Rotationally inelastic collisions}

The molecular ions can undergo inelastic collisions with neutral background-gas molecules in the experimental chamber, which lead to a loss of the originally prepared state and a redistribution of the state populations. In particular, $\mathrm{N}_{2}^{+}$ ions can undergo charge-exchange reactions with their neutral precursor molecules according to

$$
\mathrm{N}_{2}^{+}\left(N^{+}\right)+\mathrm{N}_{2}(J) \rightarrow \mathrm{N}_{2}(\bar{J})+\mathrm{N}_{2}^{+}\left(\bar{N}^{+}\right) .
$$

This process can result in the production of $\mathrm{N}_{2}^{+}$ions in different rotational states, which are subsequently recooled into the Coulomb crystal $\left(\bar{J}\right.$ and $\bar{N}^{+}$denote the rotational quantum numbers of the neutral and the ion after the collision, respectively). In order to characterize the importance of inelastic processes, we performed experiments in which the $\mathrm{N}_{2}{ }^{+}$ions were produced under collisional conditions. In Fig. 8, LICT experiments are shown in which the rotational-state populations in $N^{+}=2,3,4\left(F_{1}\right)$ were measured. The $\mathrm{N}_{2}{ }^{+}$ions were originally produced in $N^{+}=3$ by ionizing from a molecular beam passing directly through the $\mathrm{Ca}^{+}$Coulomb crystal. Thus, the sympathetically cooled $\mathrm{N}_{2}^{+}$ions were exposed to collisions with $\mathrm{N}_{2}$ molecules over the duration of the gas pulse $(1 \mathrm{~ms}$ at a repetition rate of $10 \mathrm{~Hz}$ ) during the loading period of $30 \mathrm{~s}$. The charge-transfer efficiency out of $N^{+}=3$ [Fig. 8(b)] is reduced in comparison to the experiments performed under collision-free conditions [Fig. 7(b)]. Only $4 \pm 1$ out of $24 \pm 1$ $\mathrm{N}_{2}+$ ions (17\%) were removed as opposed to an average of $27 \%$ in the experiments in which the molecular beam was offset from the trap center (see Sec. IV B). On the other hand, LICT could now be observed out of the neighboring $N^{+}=2$ and $N^{+}=4$ rotational levels [Figs. 8(a) and 8(b)] with efficiencies on the order of $\approx 1-2$ ions. We interpret this result as a clear signature of the redistribution of population caused by charge-transfer collisions of $\mathrm{N}_{2}^{+}$with $\mathrm{N}_{2}$.

The collisional redistribution of population is even more pronounced when the $\mathrm{N}_{2}{ }^{+}$ions are produced from background nitrogen gas. Under these conditions, the $\mathrm{N}_{2}^{+}$ions are exposed to the neutral gas during the entire loading period. $\mathrm{N}_{2}$ was

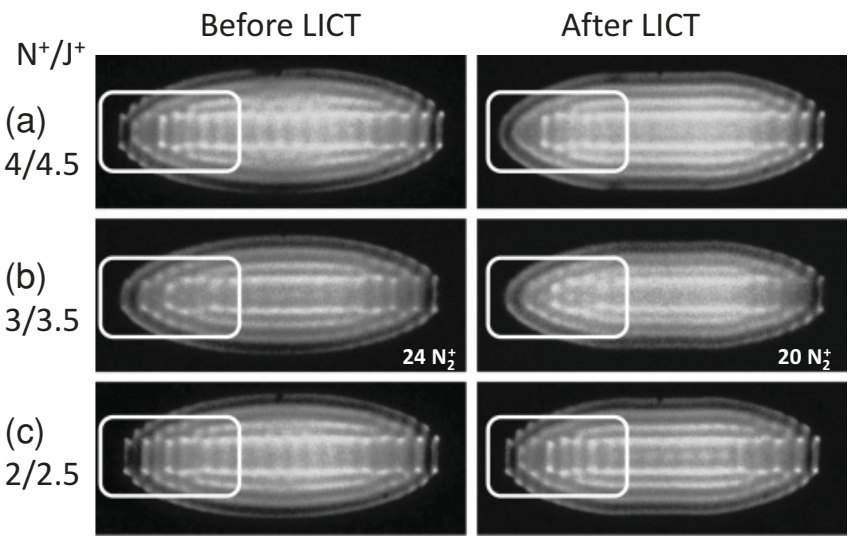

FIG. 8. LICT experiments on sympathetically cooled $\mathrm{N}_{2}^{+}$ions originally produced in $N^{+}=3$ by ionization from a molecular beam passing directly through the Coulomb crystal. The population in $N^{+}=3$ is reduced and LICT can now be observed from the neighboring $N^{+}=2,4$ levels, indicating the redistribution of population by collisional processes.
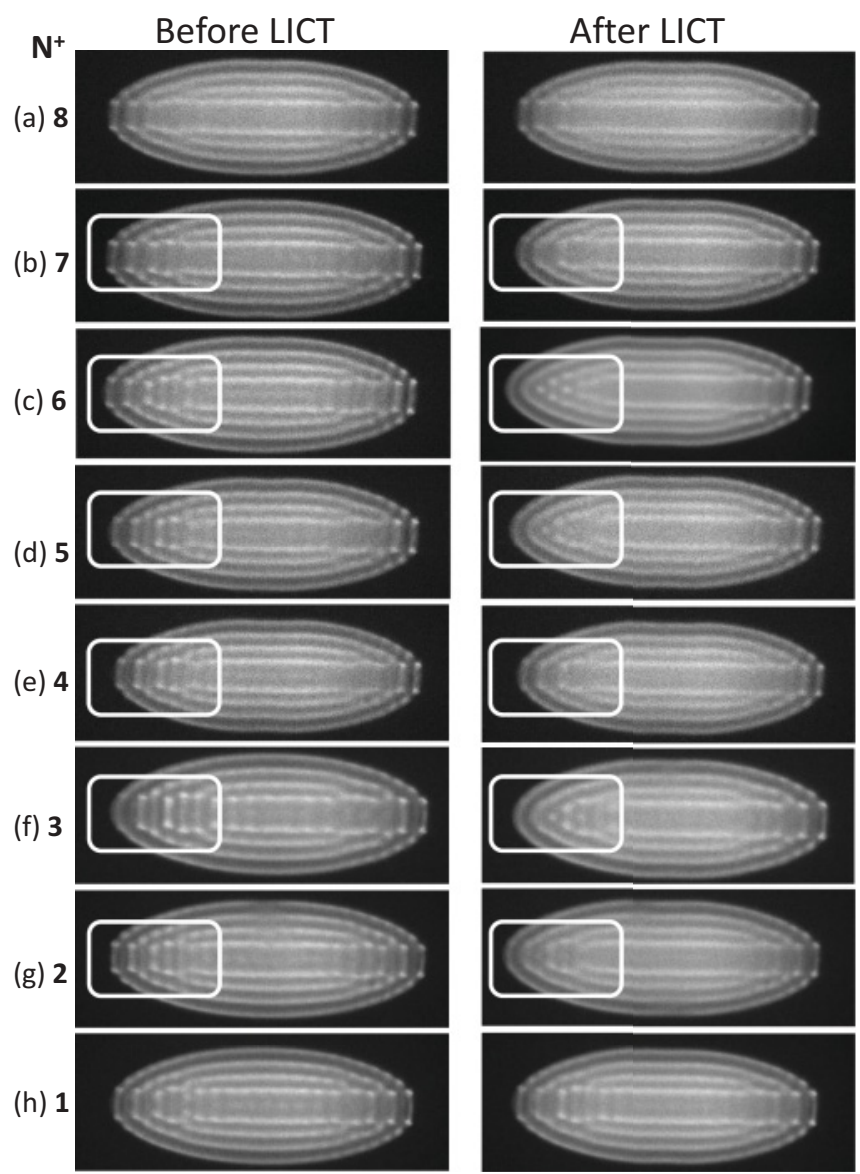

FIG. 9. LICT experiments on sympathetically cooled $\mathrm{N}_{2}^{+}$ions originally produced in $N^{+}=3$ by ionization from nitrogen background gas at a pressure of $1 \times 10^{-8}$ mbar. Under these conditions, the population is distributed over a wide range of rotational levels by inelastic collisions occurring during the loading period.

introduced into the chamber through a leak valve at a partial pressure of $1 \times 10^{-8}$ mbar for a period of $15 \mathrm{~s}$. In this case, population in levels as high as $N^{+}=7$ was detected as shown in Fig. 9. The distribution of populations observed in different rotational states is irregular, suggesting that a complete thermalization by collisions has not been achieved after $15 \mathrm{~s}$.

These results underline the importance of performing the experiments under collision-free conditions in which the production region of the ions is separated from the sympatheticcooling region. In the present study, these conditions were realized by photoionizing from a molecular beam offset from the trap center by $400 \mu \mathrm{m}$.

Under these conditions, we measure a lifetime of $\tau=$ $947 \pm 176 \mathrm{~s}$ for ions in the $N^{+}=3, F_{2}$ state [21]. We attribute the loss of population out of $N^{+}=3$ to inelastic and reactive collisions with residual background gas in the UHV chamber, in particular with $\mathrm{N}_{2}$. Assuming that the $\mathrm{N}_{2}{ }^{+}+\mathrm{N}_{2}$ charge-transfer reaction given by Eq. (1) is the do-minant inelastic loss channel of the originally prepared rotational state, we determined a second-order rate coefficient of $(3.8 \pm 2.0) \times 10^{-10} \mathrm{~cm}^{3} \mathrm{~s}^{-1}$, which is in agreement with the results reported in Ref. [38]. The bimolecular rate constant was calculated from the measured pseudo-first-order rate 
coefficient $k=\tau^{-1}$ using a nitrogen partial pressure of $p=$ $(1.1 \pm 0.6) \times 10^{-10}$ mbar in our UHV chamber, as measured with a residual-gas analyzer.

\section{Radiative heating}

Apart from inelastic collisions, radiative coupling to the ambient BBR field can lead to a redistribution of population out of the initially prepared quantum state. For apolar ions, such as $\mathrm{N}_{2}{ }^{+}$, optical transitions between rotational and vibrational levels are dipole forbidden so that radiative thermalization does not play a role on the time scale of our experiments. Consequently, the state lifetimes are determined by collisional processes (see Sec. IV C).

By contrast, for polar species, radiative coupling can constitute a major mechanism leading to the redistribution of population [40]. The time scale will in general depend on the strength of stimulated and spontaneous radiative processes. If low-lying vibrational states are present, vibrational excitations also become important. To characterize the impact of radiative heating, we implemented a generalized Einstein model for radiative coupling, as shown schematically in Fig. 10.

We have modeled the radiative redistribution of population for the prototypical polar diatomic ions $\mathrm{HCl}^{+}, \mathrm{ClF}^{+}$, and $\mathrm{BrCl}^{+}$. Because the symmetry of the electronic ground state of these molecules is ${ }^{2} \Pi_{3 / 2}$ (Hund's coupling case $a$ ), we use the total angular-momentum quantum number $J^{+}$to label the rotational energy levels. In this case, the rotational ground state is associated with the $J^{+}=\frac{3}{2}$ level [36]. Spin-forbidden transitions to the excited ${ }^{2} \Pi_{1 / 2}$ state were neglected in our radiative transfer model. Also, the $\Lambda$-type doubling of the rotational states, which has only a small influence on the level energies in the $J^{+}$range studied, was not taken into account [36].

The rate of change of the population $n_{i}$ in state $i \equiv$ $\left(v^{+}, J^{+}\right)$is given by the sum of all rates $R_{i j}$ associated with the stimulated (straight arrows) and spontaneous (wavy arrows) radiative processes between the level $i$ and all other

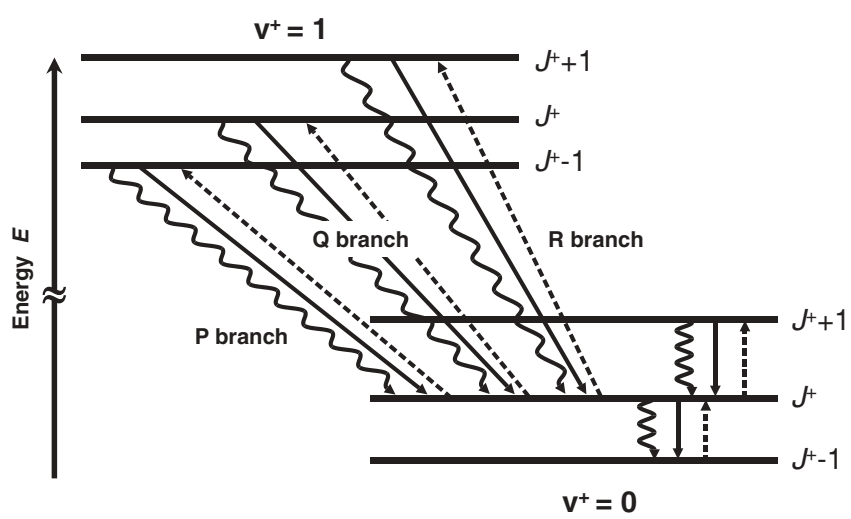

FIG. 10. Rovibrational energy-level scheme and radiative processes leading to the redistribution of population out of a rotational level $J^{+}$in the vibrational ground state by coupling to the ambient BBR field. Solid straight arrows show stimulated emission, solid wavy arrows show spontaneous emission, and dashed arrows show absorption. rotational-vibrational levels $j$ accessible by dipole-allowed transitions:

$$
\frac{d n_{i}}{d t}=\sum_{j \neq i} R_{i j}
$$

where, for level energies $E_{j}>E_{i}$,

$$
R_{i j}=-B_{i j} \rho\left(v_{i j}\right) n_{i}+B_{j i} \rho\left(v_{i j}\right) n_{j}+A_{j i} n_{j},
$$

and, for $E_{i}>E_{j}$,

$$
R_{i j}=B_{j i} \rho\left(v_{i j}\right) n_{j}-B_{i j} \rho\left(v_{i j}\right) n_{i}-A_{i j} n_{i} .
$$

Here, $B_{i j}, B_{j i}$, and $A_{j i}$ denote the Einstein coefficients for induced absorption, and stimulated and spontaneous emission [41]:

$A_{i j}=\frac{1}{g_{i}} \frac{16 \pi^{3} v_{i j}^{3}}{3 \epsilon_{0} h c^{3}}\left|D_{i j}\right|^{2}, \quad B_{i j}=\frac{c^{3}}{8 \pi h v_{i j}^{3}} A_{i j}, \quad B_{j i}=\frac{g_{i}}{g_{j}} B_{i j}$,

where the $g_{i}, g_{j}$ denote the level degeneracies. In addition, $\rho\left(v_{i j}\right)$ is the blackbody spectral-energy density at the transition frequency $v_{i j}$ given by Planck's law,

$$
\rho\left(v_{i j}\right)=\frac{8 \pi h v_{i j}^{3}}{c^{3}} \frac{1}{\exp \left\{h v_{i j} / k_{\mathrm{B}} T\right\}-1} .
$$

The transition moments $\left|D_{i j}\right|^{2}$ for pure rotational transitions $\left(i \equiv J^{+} \rightarrow j \equiv J^{+} \pm 1\right)$,

$$
\left|D_{i j}\right|^{2}=\mu^{2}\left(J^{+}+1\right),
$$

for rovibrational transitions $\left[i \equiv\left(v^{+}=0, J^{+}\right) \rightarrow j \equiv\left(v^{+}=\right.\right.$ $1, J^{+}$or $\left.\left.J^{+} \pm 1\right)\right]$,

$$
\left|D_{i j}\right|^{2}=\frac{3 c \epsilon_{0} h}{2 \pi^{2} N_{\mathrm{A}} v_{0}} A_{01} S\left(J^{+}\right),
$$

where $S\left(\mathrm{~J}^{+}\right)$denote the rotational line strength factors [36], and for the reverse processes indicated in Fig. 10 were calculated using experimental data for the molecular dipole moments $\mu$, infrared intensities $A_{01}$, and vibrational frequencies $v_{0}$ when available [39]. In cases in which experimental information was lacking, the relevant quantities were calculated $a b$ initio using the unrestricted second-order Møller-Plesset (UMP2) method and a correlation-consistent polarized valence triple zeta (cc-pVTZ) basis set implemented in the GAUSSIAN 03 program package. The data are collected in Table I. The system of coupled differential equations (2) was solved numerically. The calculated level populations were converged with respect to the number of included levels to within better than $0.001 \%$ and matched the thermal (Boltzmann-distributed) populations in equilibrium (reached at a time $t \rightarrow \infty$ ) to within better than $0.2 \%$.

For $\mathrm{HCl}^{+}, \mathrm{ClF}^{+}$, and $\mathrm{BrCl}^{+}$, the calculated time-dependent populations of the originally prepared levels $N^{+}=\frac{3}{2}$ and $\frac{5}{2}$ (corresponding to rotational ground and first excited state) are shown in Fig. 11 at two different temperatures, $T=295$ and $10 \mathrm{~K}$. Numerical results are given in Table I. For the molecules considered, the redistribution time scales and populations in thermal equilibrium vary widely and are dictated by an interplay of the magnitude of the molecular dipole moment, 
TABLE I. Molecular dipole moments $\mu$, rotational constants $B_{e}$, vibrational wave numbers $\tilde{v}_{0}$, and infrared intensities $A_{01}$ used for the modeling of radiative coupling processes and results of the numerical calculations. $\tau$ denotes the calculated radiative lifetime, $n$ is the population of the relevant rotational levels in thermal equilibrium, and "ai" stands for $a b$ initio.

\begin{tabular}{|c|c|c|c|c|c|c|c|c|c|c|c|c|c|}
\hline & \multirow{2}{*}{$\begin{array}{c}\mu \\
(\mathrm{D})\end{array}$} & \multirow{2}{*}{$\begin{array}{c}B_{e} \\
\left(\mathrm{~cm}^{-1}\right)\end{array}$} & \multirow{2}{*}{$\begin{array}{c}\tilde{v}_{0} \\
\left(\mathrm{~cm}^{-1}\right)\end{array}$} & \multirow{2}{*}{$\begin{array}{c}A_{01} \\
\left(\mathrm{~km} \mathrm{~mol}^{-1}\right)\end{array}$} & \multirow[b]{2}{*}{ Source } & \multicolumn{2}{|c|}{$\tau(295 \mathrm{~K}) / \mathrm{s}$} & \multicolumn{2}{|c|}{$\tau(10 \mathrm{~K}) / \mathrm{s}$} & \multicolumn{2}{|c|}{$n(295 \mathrm{~K}) / \%$} & \multicolumn{2}{|c|}{$n(10 \mathrm{~K}) / \%$} \\
\hline & & & & & & $J^{+}=\frac{3}{2}$ & $J^{+}=\frac{5}{2}$ & $J^{+}=\frac{3}{2}$ & $J^{+}=\frac{5}{2}$ & $J^{+}=\frac{3}{2}$ & $J^{+}=\frac{5}{2}$ & $J^{+}=\frac{3}{2}$ & $J^{+}=\frac{5}{2}$ \\
\hline $\mathrm{HCl}^{+}$ & 1.64 & 9.96 & 2674 & 2733.0 & [39], ai & 4.3 & 1.4 & 22.8 & 23.3 & 17.8 & 20.9 & 99.9 & 0.1 \\
\hline $\mathrm{ClF}^{+}$ & 2.28 & 0.59 & 1008 & 57.8 & ai & 15 & 8.5 & $1.7 \times 10^{4}$ & $6.0 \times 10^{3}$ & 3.9 & 4.1 & 29.0 & 28.5 \\
\hline $\mathrm{BrCl}^{+}$ & 0.26 & 0.17 & 624 & 6.1 & ai & 81 & 44 & $1.7 \times 10^{7}$ & $6.9 \times 10^{6}$ & 2.6 & 2.7 & 9.4 & 12.5 \\
\hline
\end{tabular}

the rotational constants, and the BBR intensity at the relevant transition frequencies [40].

The rotational populations in strongly polar ions with large rotational constants such as $\mathrm{HCl}^{+}\left(B_{e}=9.96 \mathrm{~cm}^{-1}\right)$ equilibrate on a time scale of seconds at $T=295 \mathrm{~K}$ [Fig. 11(a)]. At room temperature, the equilibrium populations are determined by optical pumping processes induced by BBR. Because of the strong dependence of the BBR intensity on the frequency $v_{i j}$ [see Eq. (6)], the efficiency of the coupling to the radiation field is determined by the position of the relevant spectroscopic transitions with respect to the BBR intensity profile. For a molecule with large rotational constants, such as $\mathrm{HCl}^{+}$, the relevant rotational transitions lie in spectral regions with relatively high BBR intensity at room temperature, leading to a strong BBR pumping rate to rotationally excited levels. At cryogenic temperatures $[T=10 \mathrm{~K}$; Fig. 11(b)], the BBR intensity is considerably reduced [see Eq. (6)] and spontaneous emission dominates the decay dynamics. Under these conditions, the population almost completely (99.9\%) accumulates in the ground rotational state in thermal equilibrium [Fig. 11(b)].

The time scales for rotational redistribution are considerably extended for molecules such as $\mathrm{BrCl}^{+}$[Figs. 11(e) and 11(f)]. The reasons for this are the smaller dipole moments leading to smaller dipole transition moments in comparison to $\mathrm{HCl}^{+}$[see Eq. (7)] and the shift of the relevant spectroscopic transitions to lower frequencies into regions of lower BBR

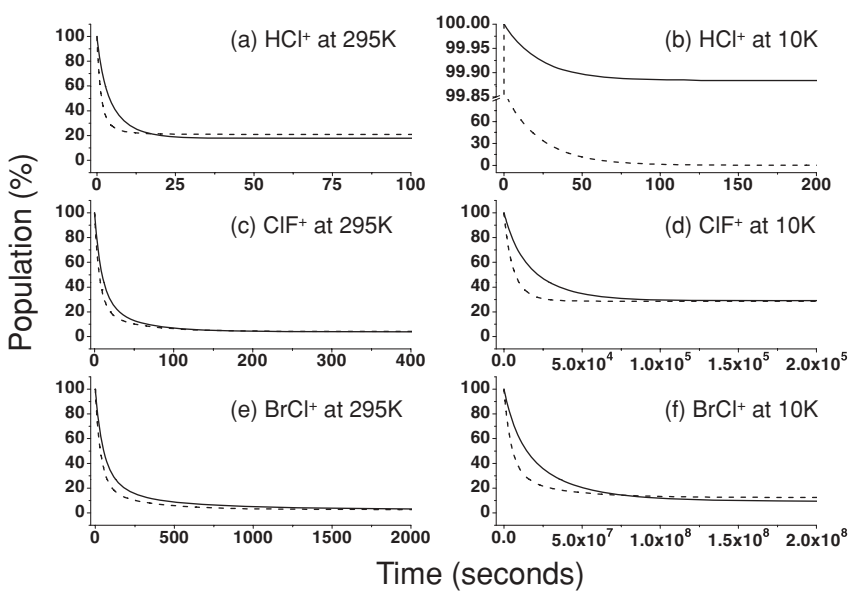

FIG. 11. Calculated decay curves of the population in the originally prepared levels $J^{+}=\frac{3}{2}$ (rotational ground state, solid lines) and $J^{+}=\frac{5}{2}$ (first excited rotational state, dashed lines) for the diatomic polar ions, $\mathrm{HCl}^{+}[(\mathrm{a}),(\mathrm{b})], \mathrm{ClF}^{+}[(\mathrm{c}),(\mathrm{d})]$, and $\mathrm{BrCl}^{+}[(\mathrm{e}),(\mathrm{f})]$, under the influence of blackbody radiation. intensity as a consequence of the small rotational constants. Moreover, for molecules with small rotational constants, spontaneous emission is a slow process because of the cubic dependence of the Einstein $A_{i j}$ coefficients on the transition frequency [see Eq. (5)] and thus plays a minor role in the population redistribution dynamics. Consequently, by cooling to cryogenic temperatures, the radiative lifetimes can be dramatically enhanced by the suppression of the stimulated processes [see Fig. 11(f)]. $\mathrm{ClF}^{+}$[see Figs. 11(c) and 11(d)] represents an intermediary case between the two limiting scenarios represented by $\mathrm{HCl}^{+}$and $\mathrm{BrCl}^{+}$.

\section{DISCUSSION}

The method for the generation of state-selected, translationally cold molecular ions presented here is widely applicable because state-selective threshold-photoionization schemes can be implemented for a broad range of molecules. A necessary prerequisite for the realization of such schemes is the precise knowledge of the rotational photoionization dynamics, which can be obtained from a rotationally resolved photoelectron spectrum. Such spectra have thus far been reported for a number of fundamentally important molecules, including $\mathrm{H}_{2}$ [42], $\mathrm{O}_{2}$ [43], $\mathrm{CO}$ [44], $\mathrm{NO}$ [45], $\mathrm{HCl}$ [46], $\mathrm{NH}_{3}$ [47], $\mathrm{C}_{2} \mathrm{H}_{4}$ [48], $\mathrm{H}_{2} \mathrm{O}$ [49], $\mathrm{CH}_{2}$ [50], $\mathrm{NH}_{2}$ [51], $\mathrm{O}_{3}$ [52], $\mathrm{CH}_{2} \mathrm{CO}$ [53], and $\mathrm{C}_{6} \mathrm{H}_{6}$ [45]. In cases in which experimental data is not available, the rotational photoionization dynamics can still approximately be inferred from theoretical considerations such as the orbital ionization model [26]. In general, optimal selectivity and flexibility in the production of the desired state is achieved with resonance-enhanced schemes which allow ionization out of a single, well-defined intermediate state, as demonstrated in the present study.

In principle, the method presented here is applicable to both polar and apolar species. The rovibrational states of apolar diatomic ions are not affected by optical dipole transitions induced by BBR. Hence it is expected that, for these ions, the state lifetimes are limited by collisional processes, as demonstrated for $\mathrm{N}_{2}{ }^{+}$in the present study. Polar ions, however, are affected by radiative heating caused by the ambient BBR field which eventually leads to a thermalization of the internalstate populations. However, even for unfavorable cases with large dipole moments and large rotational constants such as $\mathrm{HCl}^{+}$, we predict state lifetimes on the order of several seconds at room temperature, as demonstrated in Fig. 11. We expect that this time span is still sufficiently long for a wide range of different experiments. If required, even longer lifetimes 
can be achieved by cooling the trap environment to cryogenic temperatures in order to reduce the BBR intensity.

As conclusively proven in the present study, the rovibrational-state populations of the $\mathrm{N}_{2}{ }^{+}$ions are not affected by sympathetic cooling with the laser-cooled $\mathrm{Ca}^{+}$ions. According to our MD simulations, ion-ion collisions occur at typical collision distances $r$ of several hundred $\mathrm{nm}$ to $\mu \mathrm{m}$. The collisions are dominated by the long-range Coulomb interaction which scales with $r^{-1}$. This interaction is isotropic and does not couple to the internal molecular degrees of freedom. By comparison, anisotropic higher-order interactions, which can induce rotationally inelastic collisions, act on considerably shorter length scales. For $\mathrm{Ca}^{+}-\mathrm{N}_{2}^{+}$collisions, the leading anisotropic term in the interaction potential is the chargequadrupole interaction, which scales as $r^{-3}$. We conclude from our results that its influence can be neglected at the typical collision distances between the ions in our trap. For the sympathetic cooling of polar ions, the leading anisotropic-interaction term is the charge-dipole interaction, which scales with $r^{-2}$. It remains to be tested whether this interaction can induce inelastic processes under typical sympathetic-cooling conditions.

As already briefly discussed in our previous paper, the present method can be extended in several directions. Vibrationally excited states of ions can be selectively generated by ionizing through a suitable Rydberg state of the relevant neutral precursor molecule and by taking advantage of diagonal Franck-Condon factors in the photoionization step, as demonstrated in several previous studies (see, e.g., Refs. [22,23] and references therein). In combination with rotational-state selection in the Rydberg state, threshold-photoionization schemes can be devised that allow the generation of cations in well-defined excited rovibrational states. Moreover, ions in selected spin-rotational and hyperfine states can be prepared by threshold photoionization using narrow-bandwidth laser sources, as has recently been demonstrated in the case of krypton [54]. In combination with sympathetic cooling, such experiments would enable full control over all molecular degrees of freedom: translation, rotation, vibration, electronic motion, and electronic and nuclear spin.

\section{SUMMARY AND CONCLUSIONS}

We have developed a widely applicable method for the generation of internally state-selected, translationally cold molecular ions in ion traps. Our approach is based on the threshold photoionization of neutral molecules followed by the sympathetic cooling of the translational motion of the resulting ions to
mK temperatures. Using $\mathrm{N}_{2}{ }^{+}$as a test system, we demonstrated the preparation of selected rotational levels in the vibrational ground state of the ion. The internal state is fully preserved in the course of the sympathetic-cooling process and rotationalstate lifetimes on the order of 15 minutes can be achieved.

We have explored the influence of several population redistribution mechanisms on the state lifetime of the ions. For apolar ions, such as $\mathrm{N}_{2}^{+}$, the lifetimes are limited by collisional processes under our experimental conditions. For polar ions, we expect radiative processes to dominate the population redistribution dynamics. Still, our calculations show that, even in these cases, state lifetimes exceeding seconds can be achieved.

We expect that state-selected, translationally cold molecular ions will have applications in a wide range of future experiments. In combination with a source for state-selected neutral species, such as a Stark decelerator [55] or a cold-atom trap [56-58], their potential for cold ion-molecule collision studies [59] is considerable. As has recently been demonstrated for reactions of ultracold neutral molecules [8,9], a full control over the collision energy and the internal state of the reactants enables a detailed characterization of the state- and energy-dependent reaction dynamics, a long-standing goal in chemistry. Our method paves the way to extend these studies to a wide range of ion-molecule processes. Internal-state selection is also a requirement for precision measurements on single molecular ions using, e.g., quantum-logic spectroscopy $[60,61]$. Moreover, single state-selected molecular ions can form the basis of molecular-ion qubits, offering possibilities to perform quantum-information processing with molecular species along the lines of experiments already performed with atomic ions [62]. An advantage of molecular ions lies in the longevity of certain internal states (such as the rotational states in $\mathrm{N}_{2}^{+}$), which could lead to very long decoherence times, a key requirement for the implementation of complex quantum-information algorithms.

\section{ACKNOWLEDGMENTS}

This work is supported by the Swiss National Science Foundation (Grant No. PP002_118921), the National Competence Center for Research in Nanoscale Science, and the University of Basel. We thank Grischa Martin and Franz Häfeli for technical support, and Georg Holderied, Andreas Tonin, and Roberto Maffolini for electronics support. Help with the numerical modeling is acknowledged from Markus Gantenbein, Alexander Winney, and Matthias Germann.
[1] F. Lang, K. Winkler, C. Strauss, R. Grimm, and J. Hecker Denschlag, Phys. Rev. Lett. 101, 133005 (2008).

[2] K.-K. Ni, S. Ospelkaus, M. H. G. de Miranda, A. Pe'er, B. Neyenhuis, J. J. Zirbel, S. Kotochigova, P. S. Julienne, D. S. Jin, and J. Ye, Science 322, 231 (2008).

[3] J. Deiglmayr, A. Grochola, M. Repp, K. Mörtlbauer, C. Glück, J. Lange, O. Dulieu, R. Wester, and M. Weidemüller, Phys. Rev. Lett. 101, 133004 (2008).

[4] M. Vitteau, A. Chotia, M. Allegrini, N. Bouloufa, O. Dulieu, D. Comparat, and P. Pillet, Science 321, 232 (2008).
[5] S. Ospelkaus, K.-K. Ni, G. Quemener, B. Neyenhuis, D. Wang, M. H. G. de Miranda, J. L. Bohn, J. Ye, and D. S. Jin, Phys. Rev. Lett. 104, 030402 (2010).

[6] J. G. Danzl, M. J. Mark, E. Haller, M. Gustavsson, R. Hart, J. Aldegunde, J. M. Hutson, and H.-C. Nägerl, Nature Phys. 6, 265 (2010).

[7] D. DeMille, Phys. Rev. Lett. 88, 067901 (2002).

[8] K.-K. Ni, S. Ospelkaus, D. Wang, G. Quémeéner, B. Neyenhuis, M. H. G. de Miranda, J. L. Bohn, J. Ye, and D. S. Jin, Nature (London) 464, 1324 (2010). 
[9] S. Ospelkaus, K.-K. Ni, D. Wang, M. H. G. de Miranda, B. Neyenhuis, G. Quéméner, P. S. Julienne, J. L. Bohn, D. S. Jin, and J. Ye, Science 327, 853 (2010).

[10] S. Knoop, F. Ferlaino, M. Berninger, M. Mark, H.-C. Nägerl, R. Grimm, J. P. D’Incao, and B. D. Esry, Phys. Rev. Lett. 104, 053201 (2010).

[11] K. Molhave and M. Drewsen, Phys. Rev. A 62, 011401 (2000).

[12] S. Willitsch, M. T. Bell, A. Gingell, and T. P. Softley, Phys. Chem. Chem. Phys. 10, 7200 (2008).

[13] R. Wester, J. Phys. B 42, 154001 (2009).

[14] A. Bertelsen, S. Jorgensen, and M. Drewsen, J. Phys. B 39, L83 (2006).

[15] J. C. J. Koelemeij, B. Roth, and S. Schiller, Phys. Rev. A 76, 023413 (2007).

[16] I. S. Vogelius, L. B. Madsen, and M. Drewsen, Phys. Rev. Lett. 89, 173003 (2002).

[17] I. S. Vogelius, L. B. Madsen, and M. Drewsen, J. Phys. B 37, 4571 (2004).

[18] I. S. Vogelius, L. B. Madsen, and M. Drewsen, J. Phys. B 39, S1267 (2006).

[19] P. F. Staanum, K. Højbjerre, P. S. Skyt, A. K. Hansen, and M. Drewsen, Nature Phys. 6, 271 (2010).

[20] T. Schneider, B. Roth, H. Duncker, I. Ernsting, and S. Schiller, Nature Phys. 6, 275 (2010).

[21] X. Tong, A. H. Winney, and S. Willitsch, Phys. Rev. Lett. 105, 143001 (2010).

[22] S. L. Anderson, Adv. Chem. Phys. 82, 177 (1992).

[23] A. D. Sage, T. A. A. Oliver, R. N. Dixon, and M. N. R. Ashfold, Mol. Phys. 108, 945 (2010).

[24] J. Xie and R. N. Zare, J. Chem. Phys. 93, 3033 (1990).

[25] S. Opitz, D. Proch, T. Trickl, and K. L. Kompa, Chem. Phys. 143, 305 (1990).

[26] S. Willitsch and F. Merkt, Int. J. Mass Spectrom. 245, 14 (2005).

[27] M. Penno and K.-M. Weitzel, Z. Phys. Chem. 218, 311 (2004).

[28] S. R. Mackenzie and T. P. Softley, J. Chem. Phys. 101, 10609 (1994).

[29] S. R. Mackenzie, F. Merkt, E. J. Halse, and T. P. Softley, Mol. Phys. 86, 1283 (1995).

[30] R. J. Green, J. Qian, H.-T. Kim, and S. L. Anderson, J. Chem. Phys. 113, 3002 (2000).

[31] F. Merkt, Annu. Rev. Phys. Chem. 48, 675 (1997).

[32] T. F. Hanisco and A. C. Kummel, J. Phys. Chem. 95, 8565 (1991).

[33] C. B. Zhang, D. Offenberg, B. Roth, M. A. Wilson, and S. Schiller, Phys. Rev. A 76, 012719 (2007).

[34] M. Bell, A. Gingell, J. Oldham, T. P. Softley, and S. Willitsch, Faraday Discuss. 142, 73 (2009).

[35] S. Schlemmer, T. Kuhn, E. Lescop, and D. Gerlich, Int. J. Mass Spectrom. 185/186/187, 589 (1999).

[36] G. Herzberg, Spectra of Diatomic Molecules, Molecular Spectra and Molecular Structure, Vol. I (Krieger, Malabar, FL, 1989).
[37] Y.-D. Wu, J.-W. Ben, B. Li, L.-J. Zheng, Y.-Q. Chen, and X.-H. Yang, Chin. J. Chem. Phys. 20, 285 (2007).

[38] M. J. Frost, S. Kato, V. M. Bierbaum, and S. R. Leone, J. Chem. Phys. 100, 6359 (1994).

[39] K. P. Huber and G. Herzberg, Constants of Diatomic Molecules (Van Nostrand Reinhold, New York, 1979).

[40] S. Hoekstra, J. J. Gilijamse, B. Sartakov, N. Vanhaecke, L. Scharfenberg, S. Y. T. van de Meerakker, and G. Meijer, Phys. Rev. Lett. 98, 133001 (2007).

[41] W. Demtröder, Laser Spectroscopy, 4th ed. (Springer, Berlin, 2008).

[42] S. Stimson, Y.-J. Chen, M. Evans, C.-L. Liao, C. Y. Ng, C.-W. Hsu, and P. Heimann, Chem. Phys. Lett. 289, 507 (1998).

[43] F. Merkt, R. Signorell, H. Palm, A. Osterwalder, and M. Sommavilla, Mol. Phys. 95, 1045 (1998).

[44] S. R. Mackenzie, E. J. Halse, E. Gordon, R. Rolland, and T. P. Softley, Chem. Phys. 209, 127 (1996).

[45] I. Fischer, R. Lindner, and K. Müller-Dethlefs, J. Chem. Soc., Faraday Trans. 90, 2425 (1994).

[46] K. Wang and V. McKoy, J. Chem. Phys. 95, 8718 (1991).

[47] R. Seiler, U. Hollenstein, T. P. Softley, and F. Merkt, J. Chem. Phys. 118, 10024 (2003).

[48] S. Willitsch, U. Hollenstein, and F. Merkt, J. Chem. Phys. 120, 1761 (2004).

[49] M.-T. Lee, K. Wang, and V. McKoy, J. Chem. Phys. 97, 3108 (1992).

[50] S. Willitsch and F. Merkt, J. Chem. Phys. 118, 2235 (2003).

[51] S. Willitsch, Ch. Jungen, and F. Merkt, J. Chem. Phys. 124, 204312 (2006).

[52] S. Willitsch, F. Innocenti, J. M. Dyke, and F. Merkt, J. Chem. Phys. 122, 024311 (2005).

[53] S. Willitsch, A. Haldi, and F. Merkt, Chem. Phys. Lett. 353, 167 (2002).

[54] T. A. Paul, J. Liu, and F. Merkt, Phys. Rev. A 79, 022505 (2009).

[55] S. Y. T. van de Meerakker, H. L. Bethlem, and G. Meijer, Nature Phys. 4, 595 (2008).

[56] A. T. Grier, M. Cetina, F. Oručević, and V. Vuletić, Phys. Rev. Lett. 102, 223201 (2009).

[57] C. Zipkes, S. Palzer, C. Sias, and M. Köhl, Nature (London) 464, 388 (2010).

[58] S. Schmid, A. Härter, and J. Hecker Denschlag, Phys. Rev. Lett. 105, 133202 (2010).

[59] S. Willitsch, M. T. Bell, A. D. Gingell, S. R. Procter, and T. P. Softley, Phys. Rev. Lett. 100, 043203 (2008).

[60] P. O. Schmidt, T. Rosenbrand, C. Langer, W. M. Itano, J. C. Bergquist, and D. J. Wineland, Science 309, 749 (2005).

[61] P. O. Schmidt, T. Rosenband, J. C. J. Koelemeij, D. B. Hume, W. M. Itano, J. C. Bergquist, and D. J. Wineland, in AIP Conference Proceedings, edited by M. Drewsen, U. Uggerhoj, and H. Knudsen (American Institute of Physics, Melville, NY, 2006), Vol. CP862, p. 305.

[62] H. Häffner, C. F. Roos, and R. Blatt, Phys. Rep. 469, 155 (2008). 